\title{
General Psychiatry Validity and reliability of the Brain Health Self-Efficacy Scale for the elderly
}

To cite: Gao Y, Cui M, Yang $\mathrm{C}$, et al. Validity and reliability of the Brain Health Self-Efficacy Scale for the elderly. General Psychiatry 2020;33:e100208. doi:10.1136/ gpsych-2020-100208

YG and MC contributed equally.

Received 10 February 2020

Revised 24 May 2020

Accepted 05 June 2020

\section{Check for updates}

(c) Author(s) (or their employer(s)) 2020. Re-use permitted under CC BY-NC. No commercial re-use. See rights and permissions. Published by BMJ.

${ }^{1}$ Shanghai Mental Health Center, Shanghai Jiao Tong University School of Medicine, Shanghai, China

${ }^{2}$ Yinhang Community Health Center, Yangpu District,

Shanghai, China

${ }^{3}$ Shanghai Municipal Center for Disease Control and Prevention, Shanghai, China

Correspondence to Dr Xia Li;

lixia11111@sjtu.edu.cn

Dr Yinghua Yang; 18616838682@163.com

\author{
Yining Gao, ${ }^{1}$ Ming Cui, ${ }^{2}$ Chunyan Yang, ${ }^{1}$ Yuejing Wu, ${ }^{1}$ Yun Long, ${ }^{1}$ Yaopian Chen, ${ }^{1}$ \\ Hongshen Liu, ${ }^{1}$ Lin Sun, ${ }^{1}$ Yinghua Yang, ${ }^{3}$ Xia Li (D) ${ }^{1}$
}

\section{ABSTRACT}

Background With the rapid increase in ageing population, China is confronted with the daunting challenge of a growing number of patients with neurocognitive disorders (NCDs). This trend makes the maintenance of self-health and early intervention essential, highlighting the need for a tool that assesses self-efficacy of older adults in maintaining brain health or cognitive function.

Aim This study aimed to design the Brain Health SelfEfficacy Scale (BHSES) to measure elderly individuals' attitudes to NCDs, motivations and future plans for controlling risks. The psychometric properties of BHSES have been validated.

Methods Based on the current literature and relevant models, a 19-item scale was created during the first stage. A total of 660 older adults in the Yinhang community of Shanghai were included. The statistical approaches of item analysis, exploratory factor analysis (EFA), confirmatory factor analysis (CFA), criterion-related validity and reliability test were used to evaluate the quality of BHSES. In addition, the Geriatric Depression Scale (GDS) and the Self-Rating Anxiety Scale (SAS) were used as criteria to test the criterion-related validity.

Results To test item differentiation, the study adopted item analysis and excluded item 8 . Selecting a random half of the sample for EFA, the BHSES was refined to 16 items, which were categorised into the following three dimensions: 'memory belief efficacy', 'self-care efficacy' and 'future planning efficacy'. These were highly consistent with the hypothesis model. Its cumulative variance contribution rate reached $61.14 \%$, with factor loads of all items above 0.5. The three-factor model was confirmed by the remaining data through CFA. All fit indices reached the acceptable level $\left(\chi^{2}=3.045\right.$, Goodness of Fit Index $=0.898$, adjusted Goodness of Fit Index $=0.863$, Comparative Fit Index $=0.916$, Incremental Fit Index $=0.917$, Tucker-Lewis Index $=0.900$, root mean square error of approximation $=0.079$ and root mean residual=0.068). The GDS and SAS scores revealed significant correlations with the BHSES score, indicating a high criterion-related validity. The overall Cronbach's $\alpha$ coefficient was 0.793 , with the $\alpha$ coefficients' distribution of subdimensions ranging from 0.748 to 0.883 .

Conclusions The 16-item, self-compiled BHSES is a reliable and valid measurement. It could help identify older adults with potential risks for developing NCDs or with high suspicion of cognitive impairment onset in recent periods and also offer insight into tracking brain health self-efficacy in association with cognition status.

\section{INTRODUCTION}

According to the Diagnostic and Statistical Manual of Mental Disorders, Fifth Edition, neurocognitive disorders (NCDs) have been defined as a cluster of clinical syndromes mainly characterised by cognitive function impairment, such as the impairment in learning and memory, executive function, perceptual-motor function, language and attention. As the common diseases affecting the elderly who are older than 60 years, NCDs include several categories such as delirium, mild NCD (or mild cognitive impairment, MCI), major NCD (or dementia) and other cognitive disorders. ${ }^{1}$ With the fast ageing population, China now has the most cases of $\mathrm{NCDs},{ }^{2}$ with a prevalence rate of $14.71 \%$ for MCI in those older than 65 years. ${ }^{3}$ There exist currently 6 million older adults with Alzheimer's disease (AD) under a conservative statistical approach, and this number is estimated to reach 10 million by $2050 .{ }^{4}$ The costs for each patient with $\mathrm{AD}$ have reached approximately $120000 \mathrm{RMB}^{5}{ }^{5}$ which translates to a huge burden on families and the whole society. ${ }^{6}$ In 2016, The 'Healthy China 2030' Blueprint, released by the Communist Party of China Central Committee and the State Council, promoted healthy ageing and advocated self-health care. ${ }^{7}$ Early healthrelated action and detection of NCDs are critical for arresting disease progression from MCI to dementia.

At present, owing to the rapidly increasing ageing population, China is facing a high prevalence of NCDs, resulting in severe society and family burden. However, the domestic situation for NCD screening and diagnosis is still not optimistic, manifesting as a low detection rate $^{8}$ caused by the neglect and refusal of patients and their family caregivers. Older adults may not actively seek medical support in consideration of the disease discrimination, and they are not willing to conduct follow-up interventions even on receiving cognition risk prewarning from medical 
institutions. ${ }^{9}$ The main reasons for the low detection are the lack of relevant knowledge on cognitive impairment $\mathrm{t}^{10}$ and the feeling of shame associated with a confirmed diagnosis of NCDs. ${ }^{11}$ Previous surveys have shown that $45.2 \%$ of aged respondents delayed the diagnosis because they perceived dementia as a symptom of normal ageing and displayed ignorance about the disease. ${ }^{12}$ More than half of the elderly believed that they would be labelled as mentally ill after diagnosis, with the obvious stigma. As such, most older adults in China are unwilling to participate in NCD screening even if subjective cognitive decline occurs. Nevertheless, it is acknowledged that early intervention in the prodromal phase and onset stage of NCDs has clinical effectiveness. ${ }^{13}$ Early risk detection, diagnosis and treatment could delay the course of cognitive deterioration before the onset of serious dementia and also alleviate caregiver burdens from both psychological and economic perspectives. ${ }^{14}$ Relevant evidence supports that adequate self-care awareness and health-related action could reduce dementia risks by $10 \%-25 \% .{ }^{15}$ Moderate exercise, active social interaction and regular cognitive training could be seen as protective factors for retarding brain degradation. ${ }^{16}{ }^{17}$ If self-care and timely medical treatment are not taken seriously, patients with MCI who fail to be recognised at an early stage will see a rapid progression to dementia, characterised by the loss of daily function and long-term apathy. By this time, these patients with severe dementia may significantly lose selfmanagement capabilities.

Based on the actual barriers and practical significance of early screening and diagnosis, assessing the 'efficacy' of the elderly, such as attitudes towards NCDs, intentions or motivations on brain health behaviours, is particularly important for risk assessment, which could also pave the way for following targeted interventions. However, there still exists a gap with respect to the association between self-care efficacy and maintenance of cognitive health. Moreover, similar scales on selfefficacy $^{18}{ }^{19}$ such as the Chronic Disease Self-Efficacy Scale, ${ }^{20}$ self-rated abilities for health practices ${ }^{21}$ and strategies used by patients to promote health ${ }^{18}{ }^{22}$ have been widely used for physical health, but there is no specific self-efficacy assessment for NCDs, which also shows a wide area for research.

On this basis, this study raises the concept of brain health self-efficacy (BHSE). BHSE originates from the concept of 'self-efficacy' proposed by Bandura ${ }^{23}$ and can be defined as the attitude towards one's own cognitive function, as well as the motivation and confidence to maintain and improve brain health through self-care behaviour. It highlights that individuals with high selfmanagement efficacy are more likely to take steps for slowing disease progression. ${ }^{24}$

In this study, the specialised Brain Health Self-Efficacy Scale (BHSES) was compiled for the purpose of identifying older adults at risk of MCI or dementia and helping track self-efficacy related to cognition in recent times. For its development, the psychometric properties of validity and reliability were examined to ensure its applicability on a sample of older adults from the community.

\section{METHODS}

\section{Assessment tools}

The questionnaire consists of three sections: (1) general demographic data, including gender, age, ethnic group, education and so on; (2) the preliminary version of the BHSES with 19 items; and (3) measurements for assessing the criterion-related validity: the Geriatric Depression Scale (GDS-15) and the Self-Rating Anxiety Scale (SAS).

\section{Brain Health Self-Efficacy Scale Construction of BHSES}

At the early stage of BHSES development, relevant literature was taken as reference, including the literature on (1) self-efficacy, (2) self-care efficacy or health promotion, and (3) projected and controllable risk of NCDs and intervention effects targeted at these risks.

Bandura's social cognitive model, ${ }^{23}$ Pender's health promotion model $^{25}$ and Orem's self-care theory ${ }^{26}$ were used to compile the initial BHSES structure. From a macro perspective, the social cognitive model settled a firm theoretical foundation for BHSES. It proposed the concept of 'self-efficacy' and emphasised the predictive power of inner beliefs or self-care capabilities on behaviours. ${ }^{23}$ Based on this, the health promotion model and the selfcare theory paved the way for the establishment of dimension for BHSES. The health promotion model focused on personal health responsibilities and goal-directed health-promoting behaviours. It aimed to articulate and measure one's health-promoting behaviours or explicit lifestyle patterns, including nutrition, physical activities, interpersonal relationships, mental energy maintenance and so on. ${ }^{25}$ This implies that the risk factors for NCDs that could be well controlled to promote brain health should be considered in this study. In addition, the selfcare theory introduced three stages from the intention of inner self-care to outer health-related behaviours ${ }^{26}$ and provided the reference for the design of the BHSES framework. Its first stage named 'productive estimation operation' referred to one's perception and evaluation to both self and environment. Based on this, items on 'how likely to get dementia' and 'dementia to what extent influences the surrounding environment' were considered. At the 'transitional stage', individuals had perceived belief on 'whether I can, should and will do self-care operations to meet with brain health requisites', which indicated that BHSE could contain the current condition of brain health management, and future self-care expectations. Afterwards, individuals would implement health-promoting action through the 'productive self-care operation' stage.

Based on the relevant models and theories mentioned above, the preliminary scale framework contained three dimensions: (1) belief or attitude on memory, including one's subjective feeling and prediction of the likelihood of getting NCDs, the following outcomes and corresponding 
influences; (2) current brain health management, including individuals' evaluations of self-care capabilities in daily life; and (3) expectations for implementing brain healthcare, including several items asking the elderly whether they have confidence in persistently taking health-related actions during the following week.

During the item development stage, reports on the controllable potential risks of NCDs and cognitive function self-care ${ }^{152728}$ were summarised for the screening property of BHSES. It was found that high cognitive activities, ${ }^{4}$ social interactions, ${ }^{29}$ healthy $\operatorname{diet}^{30}$ and physical activities ${ }^{31}$ could be viewed as protective factors, whereas depression, ${ }^{32}$ smoking, ${ }^{33} 34$ hypertension ${ }^{35}$ and diabetes mellitus ${ }^{36}$ were targeted as risk factors. This category of elements related to brain health was incorporated as the scale content. Moreover, we took a similar measurement on self-care self-efficacy (SCSE) for reference. ${ }^{18}$

To test the rationality of the scale, five specialists, two nurses and one social worker from the department of geriatric psychiatry at the Shanghai Mental Health Center were invited to evaluate the structure and corresponding items of each dimension, and professionals in the field of public health, psychology and mental health provided suggestions to improve the quality of the scale. The study also invited five elderly people to assess each item critically, so as to check for content clarity and popularity.

BHSES is designed as a 5-point Likert-type scale, with points 1 to 5 , respectively, representing the responses of 'strongly disagree', 'partially disagree', 'neutral (neither agree nor disagree)', 'partially agree' and 'strongly agree'. Scores from 1 to 5 in turn indicate the extent of agreement with each item statement. The higher the item score, the deeper the agreement.

\section{Scale quality precontrol}

The original item pool was generated with 23 items. Through quality precontrol, modification, supplementation and refinement were made to improve the scale on the basis of multiple aspects derived from general comments on the BHSES content, scale purpose, response accuracy, language clarity, missing information and so on.

The initial item version included reverse-scored items, and we considered whether or not the BHSES would contain this category of items. Because a proportion of participants in this study were relatively low-educated, reverse-scored items might be more difficult to understand and could increase the possibility of misunderstandings. ${ }^{37}$ Researchers have found that within some dimensions, mixing reverse-scored items with positively worded items tends to cause random or systematic errors, and reduces the response reliability and validity. ${ }^{38} 39$ Therefore, these negatively worded items were modified into positively worded forms to prevent participants from misinterpreting items or forgetting to reverse ratings. For instance, 'It is hard for me to keep a balanced diet' was modified into 'I have a balanced diet'.

Taking the accuracy of the response into consideration, ratings of some items are prone to be affected by confounding factors such as inner emotional states and thought habits. The excluded item named 'I think my cognition function is declining' tends to be influenced by the individual's depression level because researchers have found a robust relationship between subjective memory decline and depression. ${ }^{40}$ 'I think my memory is worse than 10 years ago' was also excluded because of its possibility of false positives on confusion with normal ageing. Moreover, items merely targeted at controlling alcohol or smoking were removed owing to the potential response difference between male and female participants.

Some item statements needed to be revised because they were considered too vague. Phrases such as 'social interaction' and 'active mind' were too abstract for the elderly to comprehend, especially for low-educated people. They were refined to more specific and colloquial forms such as 'communicating with other', 'go out for social contact', 'learn latest knowledge or try new activities'.

After revising the items, a convenient sampling method was used to select 35 elderly people older than 60 years for the pretest. The overall Cronbach's $\alpha$ coefficient was 0.786 , which revealed the relatively high reliability. Therefore, the preliminary version of BHSES contained 19 items.

\section{Measurements for assessing criterion-related validity Geriatric Depression Scale}

This scale was first developed by Brink and Yesavage in 1982. It was suitable for assessing the extent of depression among elderly people. ${ }^{41}$ Its simplified version has 15 items, which were revised based on the original 30-item version. ${ }^{42}$ The score ranges from 0 to 15 , and a score of 8 or above represents depression. The respondents answer 'yes' or 'no' according to their recent subjective feelings. This scale has been verified with good reliability and validity among elderly people in Chinese communities with a Cronbach's $\alpha$ coefficient of $0.763{ }^{17}$

\section{Self-Rating Anxiety Scale}

SAS was first designed by Zung. Among the 20 items, 5 are worded to produce reverse scores. The scale uses 4-point Likert scoring, which in turn reflects the incremental occurrence frequency of anxiety-related behaviour samples by each item scoring from 1 to 4 points. The higher the score, the more severe the anxiety. The Cronbach's $\alpha$ coefficient of the revised Chinese version is 0.931, which could perfectly assess the extent to which the respondents feel anxious. ${ }^{43}$

\section{Sampling}

Using convenient sampling, participants were recruited from free physical check-ups organised by the Yinhang community of Yangpu District, Shanghai. A total of 1039 people participated in the free community check-ups. Participants needed to be able to read and understand the assessment content, and they also needed to accept the written informed consent. Among the excluded samples, 322 were younger than 60 years, 14 declined to 


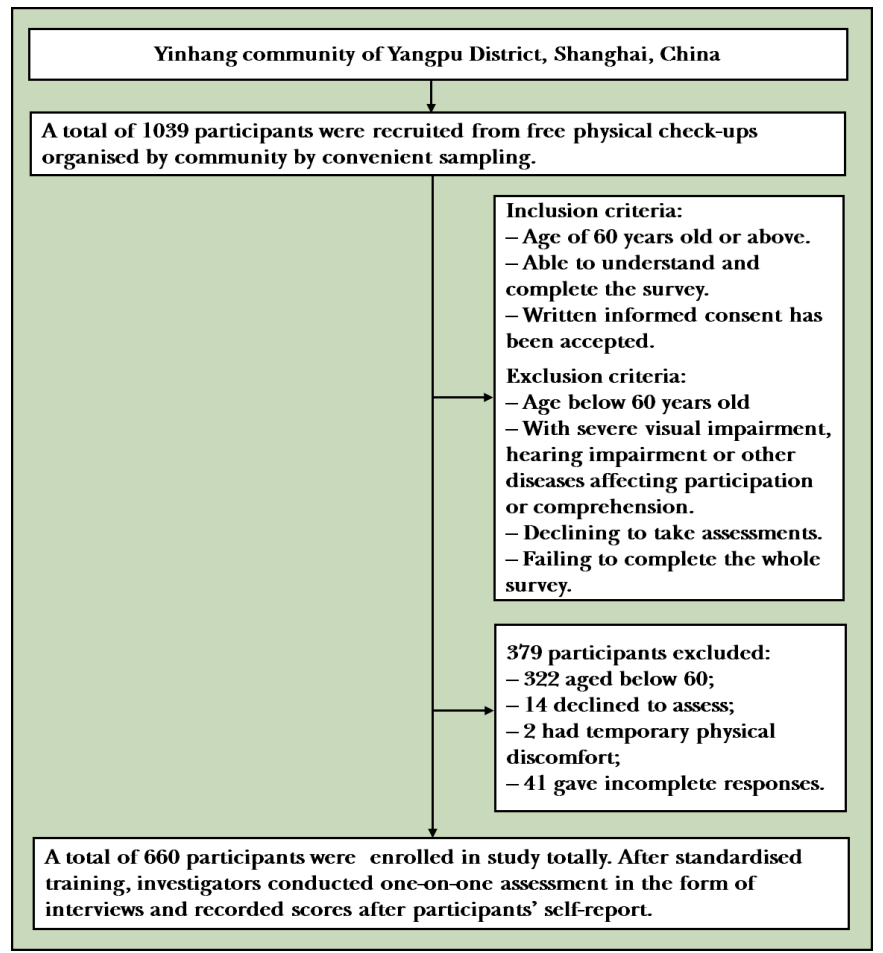

Figure 1 Participation flowchart of this study.

participate, 2 were absent owing to temporary physical discomfort and 41 gave incomplete responses. In total, 660 valid participants were enrolled in the study, with an effective recovery rate of $92.2 \%$. The study participation flowchart is shown in figure 1.

Of the 660 valid participants, demographic information was as follows: age distribution (69.91(6.75) years), with 401 aged between 60 years and 70 years $(60.8 \%), 192$ aged between 71 years and 80 years $(29.1 \%), 67$ aged above 80 years $(10.1 \%)$; male $(n=265,41.7 \%)$, female $(n=385$, $58.3 \%)$; Han ethnic group ( $\mathrm{n}=660,100 \%)$; primary school and below $(\mathrm{n}=84,12.7 \%)$, secondary school education level $(n=468,70.9 \%)$, college degree and above $(n=67$, $10.2 \%)$ and other $(n=41,6.2 \%)$.

According to the sampling theories of the structural equation model, the sample size should be 10 times greater than the number of observed variables, and the more the better. ${ }^{44}$ In this study, the preliminary scale had 19 items, so the sample size should be more than 190 . The actual size was 660 , indicating the appropriateness of the sample, in which 330 were, respectively, selected for implementing exploratory factor analysis (EFA) and confirmatory factor analysis (CFA) in random.

\section{Procedure}

The investigators were beforehand given specific and professional guidance on their assessment role, response attitude, how to properly conduct the questionnaires, types of questions and the corresponding appropriate grading method.

At the formal assessment stage, investigators were responsible for asking participants about general demographic information (eg, gender, age, ethnic group, education) and instructing participants to complete the three scales (BHSES, GDS and SAS) by one-on-one interviews so as to reflect individuals' real attitudes or mental states as truthfully and unbiasedly as possible, and avoid recording subjects' casual answers without full comprehension.

Before conducting the BHSES, the investigators first informed the participants of the scoring rules. The instructions were as follows: 'Each item is a statement, and you need to objectively judge whether it fits your recent conditions based on your daily situations, attitudes, beliefs or true thoughts. You should report in the form of numbers from 1 to 5 to express to what extent you agree with each statement. Point one represents strongly disagree; point two represents partially disagree; point three represents neutral (neither agree nor disagree); point four represents partially agree; point five represents strongly agree'. To make sure that participants understood instructions and adapted to the BHSES response mode, response examples in plain expressions were provided if possible. While assessing the BHSES, if the participants responded naturally and decisively and conformed to the rules, these would be counted as effective scores. For hesitant responses or reports of non-compliance, scoring rules would be reiterated to remind proper reactions.

At the data collection stage, investigators checked the completion quality of each participant and excluded the non-cooperative participants and low response completion with less than $80 \%$ information. The study applied Epidate V.3.1 software for double data input to ensure input accuracy.

\section{Statistical analysis}

SPSS V.17.0 software and AMOS 24.0 software were used for statistical analysis. Regarding general demographic data, mean (SD) was used for measurement data while frequency (percentage) was used for counting information. During the reliability and validity analysis, item analysis indexes (critical ratio value and item-total correlation), relevant indexes of EFA and CFA, correlation coefficients of subdimensions and the overall BHSES, correlation coefficients of criterion-related validity and Cronbach's $\alpha$ coefficients were reported. The method of allying EFA with CFA, named cross-validation, was used to ensure certainty, stability and reliability of the scale. ${ }^{45}$ AMOS is a common tool to conduct CFA by setting up the framework and validating the goodness of fit indices.

\section{RESULTS}

\section{Item analysis}

This research adopted the Critical Ratio Test (CR-test) for item analysis. After ranking the total scale score, we defined participant groups whose scores ranked from the highest to the top 27\% critical point as the 'high-score group' and those whose scores ranked from the lowest to the bottom $27 \%$ critical point as the 'low-score group'. The CR test aims to identify whether any significant 


\begin{tabular}{|c|c|c|c|c|c|c|c|}
\hline Item & Low & High & CR & Item & Low & High & CR \\
\hline 1 & $3.16(1.08)$ & 4.27 (1.05) & $-10.156^{\star * *}$ & 11 & $3.60(1.08)$ & $4.84(0.51)$ & $-14.038^{\star * *}$ \\
\hline 2 & $2.42(1.29)$ & 3.76 (1.49) & $-9.249^{\star \star \star}$ & 12 & $3.43(1.10)$ & $4.66(0.84)$ & $-12.121^{\star \star \star}$ \\
\hline 3 & $2.03(1.14)$ & $2.88(1.61)$ & $-5.944^{\star \star \star}$ & 13 & $3.43(1.12)$ & $4.75(0.70)$ & $-13.550^{\star \star *}$ \\
\hline 4 & $1.92(1.14)$ & $2.40(1.55)$ & $-3.429^{\star \star}$ & 14 & $3.65(1.06)$ & $4.95(0.22)$ & $-16.066^{\star \star \star}$ \\
\hline 5 & $3.26(1.21)$ & 3.91 (1.43) & $-4.750^{\star \star \star}$ & 15 & 3.57 (1.05) & $4.85(0.41)$ & $-15.293^{\star \star \star}$ \\
\hline 6 & $3.37(1.06)$ & $4.60(0.92)$ & $-12.050^{\star \star \star}$ & 16 & $3.20(1.08)$ & $4.86(0.48)$ & $-18.789^{\star \star \star}$ \\
\hline 7 & $3.51(1.14)$ & $4.86(0.65)$ & $-13.981^{\star * *}$ & 17 & $3.10(1.02)$ & $4.66(0.84)$ & $-16.093^{\star * *}$ \\
\hline 8 & $3.31(1.13)$ & 3.54 (1.62) & -1.614 & 18 & 3.59 (1.19) & $4.86(0.54)$ & $-12.983^{\star \star \star}$ \\
\hline 9 & $3.36(1.06)$ & $4.85(0.54)$ & $-16.985^{\star \star \star}$ & 19 & 3.55 (1.14) & $4.87(0.48)$ & $-14.446^{\star \star \star}$ \\
\hline 10 & $3.56(1.12)$ & $4.88(0.41)$ & $-14.865^{\star \star \star}$ & & & & \\
\hline
\end{tabular}

${ }^{* *} \mathrm{p}<0.01 ;{ }^{* * *} \mathrm{p}<0.001$

$\mathrm{CR}$, critical ratio; High, high-score group; Low, low-score group.

differences exist between the two groups of each single item by the method of independent-sample t-test. Item 8 ('I can control my weight to avoid obesity') was removed because of its non-significant difference between groups $(\mathrm{CR}=1.614<1.98, \mathrm{p}>0.05)$. The remaining items presented highly significant $p$ values $(p \leq 0.001)$, as shown in table 1 . Moreover, the correlations between each item and the total score were approximately higher than 0.30 and lower than $0.80(\mathrm{p}<0.01)$, indicating relatively high discrimination.

\section{Validity}

\section{Exploratory factor analysis}

This study randomly selected half of the sample for EFA. At the early stage, we evaluated data for EFA appropriateness. Kaiser-Meyer-Olkin (KMO) was 0.870 , showing sampling adequacy. The approximate $\chi^{2}$ of Bartlett's spherical test was 2823.302. Its $\mathrm{p}$ value reached a highly statistically significant level $(\mathrm{p}<0.001)$, indicating that there existed common factors in the correlation matrix. The results of both tests, the KMO and Bartlett's spherical tests, showed the data appropriateness for factor analysis. ${ }^{46}$ We conducted principal-component analysis and oblique rotation to retest the BHSES construct validity with the remaining 18 items. Factors were extracted following Kaiser criteria values that were greater than 1.0 and with the reference of the scree plot. The maximum number of convergent iterations was set as 25. Four factors were eventually identified. It was found that the cumulative variance contribution rate of four factors reached $63.111 \%$, which was much higher than the $50 \%$ criterion suggested by Streiner. ${ }^{47}$ The factor structure obtained from EFA was highly consistent with the original hypothesis model in consideration of the first three dimensions. All factor loads and communalities were basically above 0.50 . However, factor loads of items 10 and 11 were both above 0.50 on factors 1 and 3 . Considering the initial theoretical structure, both items were temporarily classified as factor 3 , which needed to be retested by the subsequent CFA. Moreover, item 5 was excluded because it was the only item belonging to factor 4 with a relatively low variance contribution rate, and at least four items are necessary for a single dimension if the scale has been clearly divided. ${ }^{48}$ Item 12 was also removed because it was classified as factor 1 , which did not conform to the original presupposition.

The remaining 16 items were further analysed by the second EFA. The latest extracted three-factor construct was consistent with the theoretical framework. Three factors accounted for $61.141 \%$ of the total variance. Factor 1 represented participants' attitude on NCDs and subjective memory belief that showed their expectations to brain health or cognitive function, labelled 'memory belief efficacy'. Factor 2 represented participants' recent self-care expectation on their own brain health, labelled 'self-care efficacy'. Factor 3 represented participants' confidence on implementing risk-avoidance behaviours in the following week, labelled 'future planning efficacy'. Three factors, respectively, accounted for $7.675 \%$, $14.875 \%$ and $38.59 \%$ of the variance. The EFA results are shown in table 2 and figure 2.

\section{Confirmatory factor analysis}

To further verify the construct validity of the threedimensional 16-item theoretical framework suggested by EFA, it was tested using the other batch of data (330 samples) by CFA through the AMOS software. The construct was retested with the three factors as potential variables and the items contained in each factor as observation variables. Data processing adopted the maximum likelihood estimation method.

The CFA results are presented in table 3. $\chi 2 / \mathrm{df}$, (adjusted) Goodness of Fit Index (GFI/AGFI), Comparative Fit Index (CFI), Incremental Fit Index (IFI), Tucker-Lewis Index (TLI), root mean square error of approximation (RMSEA) and root mean residual (RMR) are reported. 
Table 2 Exploratory factor analysis factor loads, characteristic roots and sums of squares of the Brain Health Self-Efficacy Scale (BHSES)

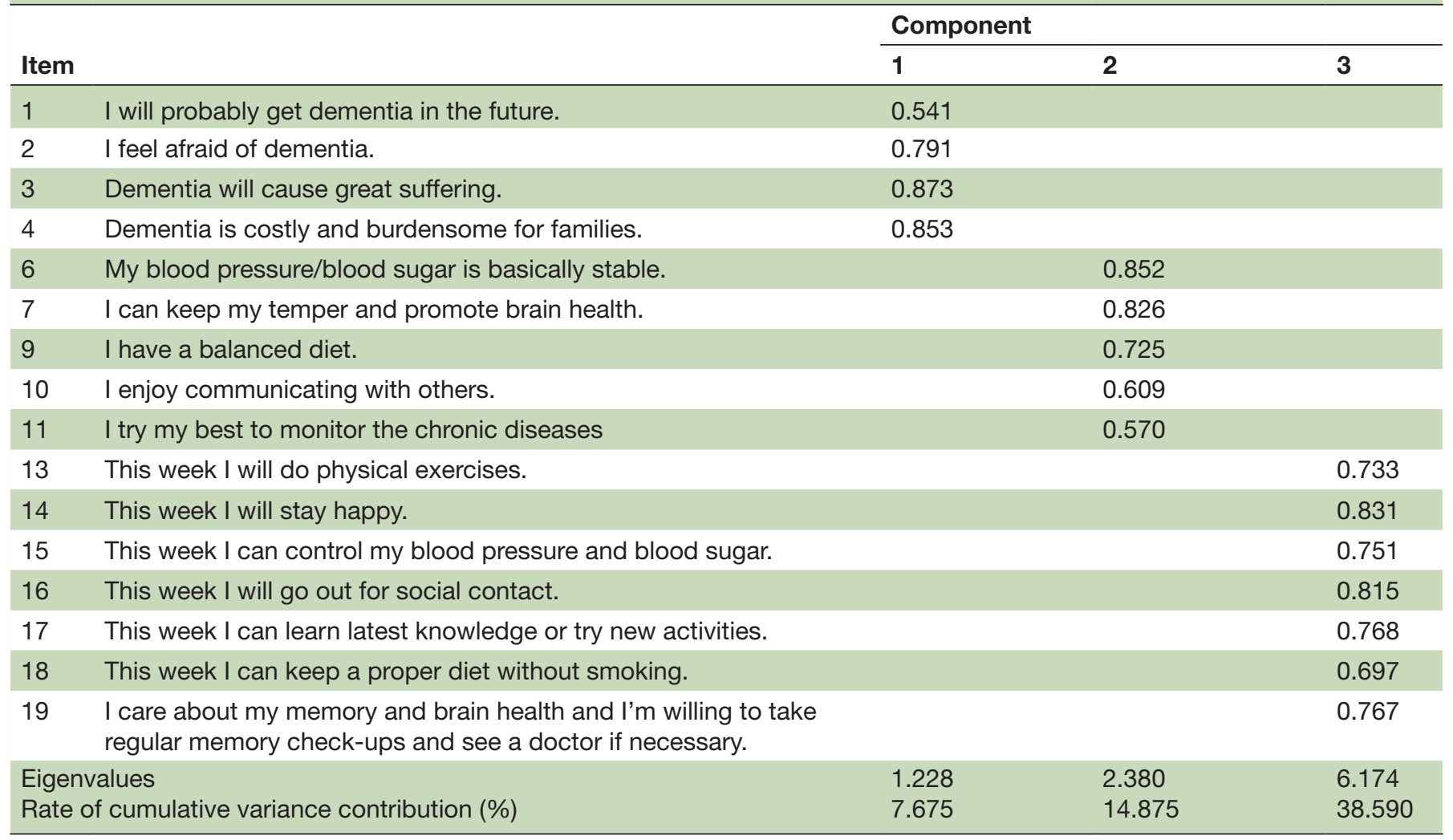

The $\chi^{2}$ represents to what degree the data accord with the hypothesised model. A $\chi^{2}$ value of $<5$ is acceptable, with a smaller value being an improvement. Among the fit indices that can compare the hypothesised scale model with other set standards, the CFI, IFI, TLI and GFI values of nearly or exceeding 0.9 are typically regarded as good fit, and AGFI $>0.8$ is acceptable. ${ }^{46} 49$ RMSEA and RMR are used to assess the model's overall fit. The RMR is seen as the residual-based index that reports differences of variance-covariance matrices between the targeted sample and the hypothesised model, whereas RMSEA can evaluate the fitness between the initial hypothesis and

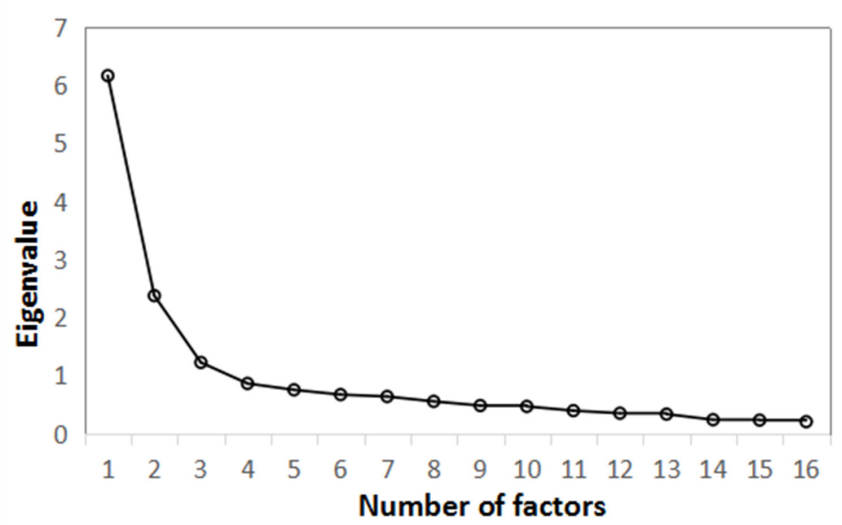

Figure 2 Scree plot for exploratory factor analysis (EFA) of the Brain Health Self-Efficacy Scale (BHSES). the population covariance matrix as a parsimony index. ${ }^{50}$ Both RMSEA and RMR values of no greater than 0.08 indicate adequate fit. ${ }^{51}$ All model-data fit indexes were within the acceptable domain that showed the results were highly consistent with the hypothetic framework.

\section{Correlations of subdimensions and the total scale}

The results in table 4 present the correlation coefficients of interdimension, and the correlation between the overall BHSES and each subdimension. It shows correlations between the overall BHSES and each subdimension were all higher than interdimension correlations, which reached a level of statistical significance.

\section{Criterion-related validity}

GDS and SAS were used as criteria to calculate their correlation coefficients with scores of the overall BHSES and subdimensions. As expected, it is shown that BHSE was conceptually related to both emotional variables that reached significance levels $(p<0.01)$. The results are shown in table 5 .

\section{Reliability}

The internal consistency of BHSES was analysed. The Cronbach's $\alpha$ reliability of the overall BHSES was 0.793 , with 0.748 for 'memory belief efficacy', 0.812 for 'self-care efficacy' and 0.833 for 'future planning efficacy'. Therefore, BHSES has high temporal stability and internal consistency. 
Table 3 Test of construct validity using confirmatory factor analysis (CFA) fit indexes of the Brain Health Self-Efficacy Scale (BHSES)

\begin{tabular}{lllllllll}
\hline Fit index & $\chi^{2 / \mathrm{df}}$ & GFI & AGFI & CFI & IFI & TLI & RMSEA & RMR \\
\hline Value & 3.045 & 0.898 & 0.863 & 0.916 & 0.917 & 0.900 & 0.079 & 0.068 \\
\hline
\end{tabular}

AGFI, adjusted Goodness of Fit Index; CFI, Comparative Fit Index; GFI, Goodness of Fit Index; IFI, Incremental Fit Index; RMR, root mean residual; RMSEA, root mean square error of approximation; TLI, Tucker-Lewis Index.

\section{DISCUSSION}

\section{Main findings}

The risk of developing NCDs increases as a function of age, and thus individuals older than 60 years are more susceptible than younger individuals. Previous studies have summarised a number of controllable dementia risks such as hyperglycaemia, hypertension, smoking, obesity, physical and cognitive inactivity, and depression. These potential risk factors could be controlled through self-care. ${ }^{15252}$ Positive BHSE is conducive to the development of a healthy mental and physical state to avoid negative dementia consequences. ${ }^{53}$ Therefore, under the background of the ageing population and the increasing number of elderly Chinese people with cognitive disorders, this study compiled BHSES. This is a specialised tool for psychological assessment of NCDs, which is pioneering in China and even the whole world.

The objective of the BHSES was to assess the beliefs of the elderly and understanding on NCDs, whether the elderly have consciousness and confidence to live by habits that are good for brain health, and whether they have plans for avoiding risks associated with cognitive impairment. BHSE was put forward based on classical models and relevant measurements on 'self-care self-efficacy' proposed earlier in medicine and nursing fields. ${ }^{18}$ Individuals' self-belief will affect their subsequent behaviours of disease management and risk avoidance in practice, so self-efficacy has been regarded as a psychological prerequisite for explicit health behaviour. It has been shown that intervention and education aimed at improving self-care efficacy could consume less resources and benefit more people in the long term. ${ }^{545}$

\begin{tabular}{|c|c|c|c|c|}
\hline & $\begin{array}{l}\text { Memory } \\
\text { belief }\end{array}$ & Self-care & $\begin{array}{l}\text { Future } \\
\text { planning }\end{array}$ & $\begin{array}{l}\text { Total } \\
\text { BHSES }\end{array}$ \\
\hline $\begin{array}{l}\text { Memory } \\
\text { belief }\end{array}$ & 1 & & & \\
\hline Self-care & $-0.126^{\star \star}$ & 1 & & \\
\hline $\begin{array}{l}\text { Future } \\
\text { planning }\end{array}$ & $-0.175^{\star \star}$ & $0.648^{* *}$ & 1 & \\
\hline Total BHSES & $0.350^{\star \star}$ & $0.742^{\star \star}$ & $0.763^{* \star}$ & 1 \\
\hline
\end{tabular}

\footnotetext{
${ }^{* *} \mathrm{p}<0.01$
}

Future planning, Future planning efficacy; Memory belief, Memory belief efficacy; Self-care, Self-care efficacy.
The total BHSES is divided into three subdimensions of 'memory belief efficacy', 'self-care efficacy' and 'future planning efficacy'. The validity of the BHSES has been proven appropriate through the method of crossvalidation including both the EFA and CFA. ${ }^{45}$

Faced with health problems, optimistic attitudes could significantly and positively predict subsequent health behaviours and strategy implementation. ${ }^{56}$ The elderly with more positive mental states reported more positive SCSE. ${ }^{57}$ This revealed the impact of emotions on efficacy and subsequent behaviour. Therefore, in this study, depression and anxiety were used as criteria variables, and both the GDS and SAS were adopted. It was found that there existed a significant negative correlation between emotions of depression and anxiety and BHSES, indicating the high criterion-related validity of BHSES.

From the perspective of reliability, this study conducted internal consistency analysis on BHSES. The Cronbach's $\alpha$ coefficient of the overall BHSES was 0.793 , and the coefficient of distribution of subdimensions was $0.748-0.883$, indicating the scale's reliability.

Given the good psychological properties of BHSES, it could be applied to the elderly Chinese for screening people with potential risk of NCDs and also provide strong evidence and guidance for the future work of early referral, targeted adjustment on healthy lifestyle and timely supervision of cognitive functions of the elderly in communities.

\section{Limitations}

Although the sample size of this study was sufficient and the quality of BHSES seemed to be good, there still existed some limitations.

First, from the perspective of sampling, the samples were the elderly individuals who voluntarily participated in the free physical examination organised by the community. This category is inclined to pay more attention to self-care and have a more positive attitude towards life. The results from 660 participants also reported that BHSE among elderly participants was relatively positive, with a total score of 62.30 (8.66). Besides, most participants came from cities $(\mathrm{n}=568,86.1 \%)$, which might not represent the overall condition among the Chinese elderly. Therefore, the sampling of subsequent research could be expanded to a wider population for further verification.

Second, the scale was administered in the form of interviews and scored by participants' self-report. This may somewhat result in a loss of objectivity; therefore, 
Table 5 Correlation coefficients between brain health self-efficacy (BHSE) and emotions of depression and anxiety

\begin{tabular}{lllll}
\hline & Memory belief & Self-care & Future planning & Total BHSES \\
\hline GDS score & $-0.082^{\star}$ & $-0.227^{\star \star}$ & $-0.226^{\star *}$ & $-0.288^{\star \star}$ \\
SAS score & -0.045 & $-0.248^{\star \star}$ & $-0.201^{\star \star}$ & $-0.245^{\star \star}$ \\
\hline
\end{tabular}

${ }^{*} \mathrm{p}<0.05 ;{ }^{* *} \mathrm{p}<0.01$

Memory belief, Memory belief efficacy; BHSES, Brain Health Self-Efficacy Scale; GDS, Geriatric Depression Scale; Future planning, Future planning efficacy; SAS, Self-Rating Anxiety Scale; Self-care, Selfcare efficacy.

informants of the participants could be recruited to set up retests on BHSES to examine authenticity of the responses. The informants should be familiar with the older adults' daily status, attitudes and health habits. If each result of informant assessments is relatively consistent with each self-reported answer of the elderly, it will be considered as a validated questionnaire.

Third, considering that participants are aged, this study might neglect their underlying diseases, which were likely to interfere with their behaviour of implementing dementia risk control from a non-psychological perspective.

\section{Implications}

BHSES possesses good reliability and validity among elderly Chinese individuals. The BHSES application values reflect identification of older adults at risk of developing MCI or dementia, screening for the ones with high suspicion of recent onset of cognitive impairment and helping track self-efficacy factors in association with cognitive health status. On this basis, BHSES could lay the foundation for further work of prevention, diagnosis and treatment of NCDs in Chinese communities and offer a window of opportunity for follow-up research on the early preventive interventions of improving cognition or reducing risks before causing the balance irremediable shifts towards brain health degradation.

Contributors $\mathrm{YG}$ and $\mathrm{SZ}$ analysed the data and drafted the manuscript. $\mathrm{XL}$ contributed to the study concept and design. MC, CY, YW, YL, YC, HL and LS collected the data. YY provided key comments and suggestions. All authors read and approved the final manuscript.

Funding This work was supported by grants from the National Key R\&D Program of China (No. 2017YFC1310501) and the National Natural Science Foundation Committee (No. 81671402).

Competing interests None declared.

Patient consent for publication Not required.

Ethics approval The study was approved by the institutional review board of the Shanghai Mental Health Center (2014-28).

Provenance and peer review Not commissioned; externally peer reviewed.

Data availability statement № additional data are available.

Open access This is an open access article distributed in accordance with the Creative Commons Attribution Non Commercial (CC BY-NC 4.0) license, which permits others to distribute, remix, adapt, build upon this work non-commercially, and license their derivative works on different terms, provided the original work is properly cited, appropriate credit is given, any changes made indicated, and the use is non-commercial. See: http://creativecommons.org/licenses/by-nc/4.0/.

\section{ORCID iD}

Xia Li http://orcid.org/0000-0003-3531-0861

\section{REFERENCES}

1 American Psychiatric Association. Diagnostic and statistical manual of mental disorders, fifth edition (DSM-5). Arlington, Texas: American Psychiatric Association, 2013.

2 Chan KY, Wang W, Wu JJ, et al. Epidemiology of Alzheimer's disease and other forms of dementia in China, 1990-2010: a systematic review and analysis. The Lancet 2013;381:2016-23.

3 Xue J, Li J, Liang J, et al. The prevalence of mild cognitive impairment in China: a systematic review. Aging Dis 2018;9:706-15.

4 Wang $\mathrm{T}$, Xiao S, Chen K, et al. Prevalence, incidence, risk and protective factors of amnestic mild cognitive impairment in the elderly in Shanghai. Curr Alzheimer Res 2017;14:460-6.

5 Jia J, Wei C, Chen S, et al. The cost of Alzheimer's disease in China and re-estimation of costs worldwide. Alzheimers Dement 2018;14:483-91.

6 Qian SX, Qiu JY, Li X. Hierarchical process to screen and diagnose neurocognitive disorders in China. Journal of Chongqing Medical University 2019;44:397-400.

7 Zeng Z, Liu J. The outline of 'Healthy China 2030' issued by the State Council of the CPC Central Committee. Bulletin of the state council of the People's Republic of China 2016:5-20.

8 Zhang Z, Chen X, Liu X. Health care situation of dementia patients in Beijing, Xi' an, Shanghai and Chengdu regions. Chin Acad Med Sci Univ 2004;26:116-21.

9 Koch T, lliffe S, EVIDEM-ED project. Rapid appraisal of barriers to the diagnosis and management of patients with dementia in primary care: a systematic review. BMC Fam Pract 2010;11:52.

10 Schwalen S, Förstl H. [Alzheimer's disease: knowledge and attitudes in a representative survey]. Neuropsychiatr 2008;22:35-7.

11 Li X, Fang W, Su N, et al. Survey in Shanghai communities: the public awareness of and attitude towards dementia. Psychogeriatrics 2011;11:83-9.

12 Iliffe S, De Lepeleire J, Van Hout H, et al. Understanding obstacles to the recognition of and response to dementia in different European countries: a modified focus group approach using multinational, multi-disciplinary expert groups. Aging Ment Health 2005;9:1-6.

13 Neumann PJ, Hermann RC, Kuntz KM, et al. Cost-effectiveness of donepezil in the treatment of mild or moderate Alzheimer's disease. Neurology 1999;52:1138-45.

14 Relkin N. Screening and early diagnosis of dementia. Am J Manag Care 2000;6:S1111-8.

15 Barnes DE, Yaffe K. The projected effect of risk factor reduction on Alzheimer's disease prevalence. Lancet Neurol 2011;10:819-28.

16 Wang $\mathrm{H}-\mathrm{X}$, Karp A, Winblad B, et al. Late-life engagement in social and leisure activities is associated with a decreased risk of dementia: a longitudinal study from the Kungsholmen project. Am J Epidemiol 2002;155:1081-7.

17 Reimers C, Knapp G, Tettenborn B. Impact of physical activity on cognition. Can physical activity prevent dementia? Aktuel Neurol 2012;39:276-91.

18 Lev EL, Owen SV. A measure of self-care self-efficacy. Res Nurs Health 1996;19:421-9.

19 Luo H, Xie B. Self-management model and its application in mental health services. Gen Psychiatry 2004:117-9.

20 Lorig KR, Sobel DS, Ritter PL, et al. Effect of a self-management program on patients with chronic disease. Eff Clin Pract 2001;4:256-62.

21 Callaghan DM. Health-promoting self-care behaviors, self-care selfefficacy, and self-care agency. Nurs Sci Q 2003;16:247-54.

22 Qian HJ, Yuan CR. The reliability and validity of the Chinese version of strategies used by people to promote health (SUPPH). Chinese Journal of Nursing 2011;45:87-9.

23 Bandura A, Freeman WH, Lightsey R. Self-efficacy: the exercise of control. J Cogn Psychother 1999;13:158-66.

24 Von Korff M, Korff V M. Collaborative management of chronic illness. Ann Intern Med 1997;127:1097-102. 
25 Pender NJ. Health promotion in nursing practice. Stamford, CT: Appleton \& Lange, 1996.

26 Orem DE. Nursing: concepts of practice. 6th edn. St. Louis: Mosby, 2001.

27 Li X, Du X, Chen TY. Main approaches to promote cognitive well-being of the elderly: a review. Chinese Mental Health Journal 2014;28:125-32.

28 Walters K, Hardoon S, Petersen I, et al. Predicting dementia risk in primary care: development and validation of the dementia risk score using routinely collected data. BMC Med 2016;14:6.

29 Verghese J, LeValley A, Derby C, et al. Leisure activities and the risk of amnestic mild cognitive impairment in the elderly. Neurology 2006;66:821-7.

30 Roberts RO, Geda YE, Cerhan JR, et al. Vegetables, unsaturated fats, moderate alcohol intake, and mild cognitive impairment. Dement Geriatr Cogn Disord 2010;29:413-23.

31 Geda YE, Roberts RO, Knopman DS, et al. Physical exercise, aging, and mild cognitive impairment: a population-based study. Arch Neurol 2010;67:80.

32 Song D, Li PWC, Yu DSF. The association between depression and mild cognitive impairment: a cross-sectional study. Int J Geriatr Psychiatry 2018;33:672-4.

33 Reitz C, den Heijer T, van Duijn C, et al. Relation between smoking and risk of dementia and Alzheimer disease: the Rotterdam study. Neurology 2007;69:998-1005.

34 Rusanen M, Kivipelto M, Quesenberry CP, et al. Heavy smoking in midlife and long-term risk of Alzheimer disease and vascular dementia. Arch Intern Med 2011;171:333-9.

35 Tervo S, Kivipelto M, Hänninen T, et al. Incidence and risk factors for mild cognitive impairment: a population-based three-year follow-up study of cognitively healthy elderly subjects. Dement Geriatr Cogn Disord 2004;17:196-203.

36 Eshkoor SA, Hamid TA, Mun CY, et al. Mild cognitive impairment and its management in older people. Clin Interv Aging 2015;10:687-93.

37 Lietz P, Lietz P. Research into questionnaire design: a summary of the literature. Int J Mark Res 2010;52:249-72.

38 Hinkin TR. A review of scale development practices in the study of organizations. J Manage 1995;21:967-88.

39 Hinkin TR. A brief tutorial on the development of measures for use in survey questionnaires. Organ Res Methods 1998;1:104-21.

40 Paradise MB, Glozier NS, Naismith SL, et al. Subjective memory complaints, vascular risk factors and psychological distress in the middle-aged: a cross-sectional study. BMC Psychiatry 2011;11:108.
41 Cheng S-T, Chan ACM. Comparative performance of long and short forms of the geriatric depression scale in mildly demented Chinese. Int J Geriatr Psychiatry 2005;20:1131-7.

42 Sheikh J. Geriatric depression scale (GDS): recent evidence and development of a shorter version. Clin Gerontol 1986;5:165-73.

43 Tao M, Gao J. Reliability and validity of the revised anxiety self-rating scale (SAS-CR). Chinese Journal of Nervous and Mental Diseases 1994:301-3.

44 Dai $X Y$, Cao YW. Some defects in development and revision of psychological rating scales. Chinese Journal of Clinical Psychology 2009;17:562-5.

45 Anderson JC, Gerbing DW. Structural equation modeling in practice: a review and recommended two-step approach. Psychol Bull 1998;103:411-23.

46 Tinsley HE, Tinsley DJ. Uses of factor analysis in counseling psychology research. J Couns Psychol 1987;34:414-24.

47 Streiner DL. Figuring out factors: the use and misuse of factor analysis. Can J Psychiatry 1994;39:135-40.

48 Fabrigar LR, Wegener DT, MacCallum RC, et al. Evaluating the use of exploratory factor analysis in psychological research. Psychol Methods 1999;4:272-99.

49 Kline RB. Principles and practice of structural equations modeling. 2nd ed. New York, NY: Guilford Press, 2005.

50 Steiger JH. Structural model evaluation and modification: an interval estimation approach. Multivariate Behav Res 1990;25:173-80.

$51 \mathrm{Hu}$ LT, Bentler PM, Kano Y. Can test statistics in covariance structure analysis be trusted? Psychol Bull 1992;112:351-62.

52 Anstey KJ, Cherbuin N, Herath PM. Development of a new method for assessing global risk of Alzheimer's disease for use in population health approaches to prevention. Prev Sci 2013;14:411-21.

53 Mak WWS, Law RW, Woo J, et al. Social support and psychological adjustment to SARS: the mediating role of self-care self-efficacy. Psychol Health 2009;24:161-74.

54 Eller LS, Lev EL, Yuan C, et al. Describing self-care self-efficacy: definition, measurement, outcomes, and implications. Int J Nurs Knowl 2018;29:38-48.

55 Kennedy A, Reeves D, Bower P, et al. The effectiveness and cost effectiveness of a national lay-led self care support programme for patients with long-term conditions: a pragmatic randomised controlled trial. J Epidemiol Community Health 2007;61:254-61.

56 Mulkana SS, Hailey BJ. The role of optimism in health-enhancing behavior. Am J Health Behav 2001;25:388-95.

57 Konopack JF, McAuley E. Efficacy-mediated effects of spirituality and physical activity on quality of life: a path analysis. Health Qual Life Outcomes 2012;10:57

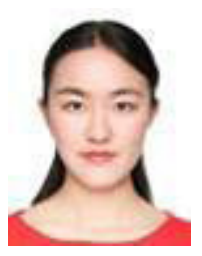

Yining Gao graduated with a bachelor's degree from East China Normal University in 2019. In the same year, she began her postgraduate studies at the Department of Geriatrics in the Shanghai Mental Health Center, Shanghai Jiao Tong University School of Medicine. She majors in clinical psychology, and her research interest includes cognitive and emotional disorders of the elderly. 